\title{
Head Motion Parameters in fMRI Differ Between Patients with Mild Cognitive Impairment and Alzheimer Disease Versus Elderly Control Subjects
}

\author{
Sven Haller • Andreas U. Monsch · Jonas Richiardi • \\ Frederik Barkhof $\cdot$ Reto W. Kressig $\cdot$ Ernst W. Radue
}

Received: 14 January 2014/ Accepted: 19 February 2014/Published online: 6 March 2014

(C) Springer Science+Business Media New York 2014

\begin{abstract}
Motion artifacts are a well-known and frequent limitation during neuroimaging workup of cognitive decline. While head motion typically deteriorates image quality, we test the hypothesis that head motion differs systematically between healthy controls (HC), amnestic mild cognitive impairment (aMCI) and Alzheimer disease (AD) and consequently might contain diagnostic information. This prospective study was approved by the local ethics committee and includes $28 \mathrm{HC}$ (age $71.0 \pm 6.9$ years, 18 females), 15 aMCI (age $67.7 \pm 10.9$ years, 9 females) and $20 \mathrm{AD}$ (age $73.4 \pm 6.8$ years, 10 females). Functional magnetic resonance imaging (fMRI) at 3T included a 9 min echo-planar imaging sequence with 180 repetitions. Cumulative average head rotation and translation was estimated based on standard fMRI preprocessing and compared between groups using receiver operating characteristic statistics. Global
\end{abstract}

\section{S. Haller $(\square)$}

Service Neuro-diagnostique et Neuro-interventionnel, Department of Imaging and Medical Informatics, University Hospitals of Geneva, Rue Gabrielle Perret-Gentil 4,

1211 Geneva 14, Switzerland

e-mail: sven.haller@hcuge.ch

\section{A. U. Monsch}

Memory Clinic, University Center for Medicine of Aging Basel, Felix Platter Hospital, Basel, Switzerland

\section{J. Richiardi}

Functional Imaging in Neuropsychiatric Disorders Laboratory, Department of Neurology and Neurological Sciences, Stanford University, Stanford, CA, USA

\section{J. Richiardi}

Laboratory for NeuroImaging of Cognition, Department of Neurosciences and Departement of Neurology, University of Geneva, Geneva, Switzerland cumulative head rotation discriminated aMCI from controls $[p<0.01$, area under curve (AUC) 0.74] and AD from controls $(p<0.01$, AUC 0.73$)$. The ratio of rotation $\mathrm{z}$ versus y discriminated $\mathrm{AD}$ from controls $(p<0.05$, AUC 0.71$)$ and $\mathrm{AD}$ from aMCI $(p<0.05$, AUC of 0.75$)$. Head motion systematically differs between aMCI/AD and controls. Since motion is not random but convoluted with diagnosis, the higher amount of motion in aMCI and $\mathrm{AD}$ as compared to controls might be a potential confounding factor for fMRI group comparisons. Additionally, head motion not only deteriorates image quality, yet also contains useful discriminatory information and is available for free as a "side product" of fMRI data preprocessing.

Keywords AD $\cdot$ MCI $\cdot$ Dementia $\cdot$ MRI $\cdot$ FMRI . Motion $\cdot$ Head motion

\footnotetext{
F. Barkhof

Department of Radiology and Nuclear Medicine, Neuroscience Campus Amsterdam, VU University Medical Centre,

Amsterdam, the Netherlands

R. W. Kressig

University Center for Medicine of Aging Basel, Felix Platter Hospital, Basel, Switzerland

E. W. Radue

Medical Image Analysis Center MIAC, University Hospitals of Basel, Basel, Switzerland
} 


$\begin{array}{ll}\text { Abbreviations } \\ \text { AD } & \text { Alzheimer disease } \\ \text { aMCI } & \text { Amnestic mild cognitive impairment } \\ \text { AUC } & \text { Area under the curve } \\ \text { fMRI } & \text { Functional magnetic resonance imaging } \\ \text { HC } & \text { Healthy control } \\ \text { MMS } & \text { Mini mental state } \\ \text { MRI } & \text { Magnetic resonance imaging } \\ \text { PET } & \text { Positron emission tomography } \\ \text { rs-fMRI } & \text { Resting-state fMRI } \\ \text { ROC } & \text { Receiver operating characteristic }\end{array}$

\section{Introduction}

Head motion related artifacts are a well-known and frequent problem in the neuroimaging workup of cognitive decline deteriorating image quality (Ikari et al. 2012; Sperling 2011). We assessed whether head motion systematically differs between amnestic mild cognitive impairment (aMCI) and Alzheimer disease (AD) patients as compared to healthy controls (HC). A potential systematic difference in head motion between groups would in turn imply that head motion does not only deteriorate image quality, but may actually contain useful discriminatory information.

The early detection of Alzheimer disease (AD) and in particular amnestic mild cognitive impairment (aMCI) is a key clinical question, as promising therapeutic candidates are currently being evaluated (for review see (Nitsch and Hock 2008; Duara et al. 2009)). Recent studies indicate a limited performance of these therapeutic candidates in patients with clinically overt dementia (Holmes et al. 2008; Lannfelt et al. 2008). This implies a need for early treatment, which in turn implies a need for early diagnosis. Mild cognitive impairment (MCI) represents a transition zone between normal aging and very early dementia and is characterized by selective memory deficits associated or not with other cognitive dysfunctions (Petersen and Negash 2008). The definitions of MCI have substantially evolved and changed over the last years, which goes beyond the scope of this manuscript. We specifically focus on amnestic MCI (aMCI), which is the most likely MCI subgroup to eventually progress to clinically overt $\mathrm{AD}$ with an annual rate of 10-15\% (Petersen 2004; Mariani et al. 2007), whereas other MCI subgroups may remain stable or evolve to other forms of dementia (Forlenza et al. 2009).

Multiple recent investigations assessed the performance of various neuroimaging techniques for the early detection of $\mathrm{AD}$ and $\mathrm{MCI}$, including $\mathrm{T} 1$ derived grey matter voxel based morphometry (Plant et al. 2010; Misra et al. 2009;
Fan et al. 2008), diffusion tensor imaging (DTI) derived white matter properties (Haller et al. 2010b, 2013; O'Dwyer et al. 2012), susceptibility derived iron deposition (Haller et al. 2010a) and in particular functional MRI (Mueller et al. 2011; Fox and Greicius 2010; Sperling 2011).

Head motion parameters are routinely estimated during the data preprocessing steps of fMRI. These parameters are typically used as non-explanatory co-variables for the data analysis, yet are not specifically analyzed as discriminatory parameters. In the current investigation, we tested the hypothesis that aMCI and AD have increased head motion parameters compared to HC. This analysis makes use of already existing and thus freely available motion correction parameters of fMRI data and might complement the results of the "actual" fMRI analyses.

\section{Materials and Methods}

\section{Participants}

The institutional ethical committee of the University of Basel, Switzerland, approved this prospective study and all participants gave written informed consent prior to inclusion. The study is in agreement with the Declaration of Helsinki. All individuals were examined as described in detail before (Monsch and Kressig 2010). The diagnosis criteria for $\mathrm{AD}$ were based on the NINCDS-ADRDA criteria (McKhann et al. 1984). The diagnosic criteria for aMCI were based on the criteria by Winblad et al. (2004). Healthy controls were recruited from the Basel Study on the Elderly. They essentially underwent the same procedure as described above (Monsch and Kressig 2010). The local memory clinic prospectively and consecutively included 63 participants.

\section{MR Imaging}

MR imaging was performed on a 3T clinical routine whole body MRI scanner (Verio, Siemens Medical Systems Erlangen, Germany). Standard routine clinical imaging included a 3DT1w (1mm3 isometric, $256 \times 256 \times 176$ matrix), DTI (30 directions, b 1000). Additional sequences (T1w, T2w, T2*, FLAIR) were acquired and analyzed to exclude brain pathology such as ischemic stroke, subdural hematomas or space-occupying lesions. T2 lesions were analyzed using a simple visual rating scale of Fazekas (Fazekas et al. 1987).

The 9 min fMRI echo planar imaging (EPI) scan covering the entire brain was acquired with the following parameters: $64 \times 48$ matrix, 34 slices, voxel size $3.44 \times 3.44 \times 3.5 \mathrm{~mm} 3$, TE $12.3,29.5,46.8$ and $64 \mathrm{~ms}$, TR 2,970 ms, 180 repetitions. 


\section{MR Data Analysis}

Data was preprocessed using standard parameters in SPM8. All functional images were motion-corrected via realignment to the mean image. The absolute values of the instantaneous differences between motion correction parameters for translation $(\mathrm{X}, \mathrm{Y}$ and $\mathrm{Z}$ ) and rotation (pitch $\mathrm{X}$, roll $\mathrm{Y}$ and yaw $\mathrm{Z}$ ) were computed and summed across the entire functional run per participant, yielding total instantaneous translation in $\mathrm{mm}$ for the three translations and radians for the three rotations. In the following, we consider cumulative head motion parameters (i.e. the sum of head motion over the 9 min acquisition time) for head rotation $(\mathrm{x}, \mathrm{y}, \mathrm{z})$ and head translation $(\mathrm{x}, \mathrm{y}, \mathrm{z})$.

\section{Statistical Analysis}

Statistical analysis was performed in Graphpad Prism, Version 5.0 (www.graphpad.com).

\section{Results}

\section{Demographic and Clinical Data}

We included $28 \mathrm{HC}$ (age $71.0 \pm 6.9$ years, 18 females, mini mental state MMS $29.2 \pm 1.0$ ), 15 aMCI (age $67.7 \pm 10.9$ years, 9 females, MMS $27.8 \pm 1.5$ ) and 20 AD (age $73.4 \pm 6.8$ years, 10 females, $24.6 \pm 3.1$ ). There was no significant difference in age (parametric ANOVA) or gender (non-parametric Kruskal-Wallis test) between groups. Likewise, there was no significant difference (nonparametric Kruskal-Wallis test) between groups with respect to (Fazekas et al. 1987) T2 lesions score (HC $1.2 \pm 0.9$, aMCI $1.1 \pm 0.8, \mathrm{AD} 1.2 \pm 0.9$ ).

In contrast, there was a significant group difference in the MMS (analysis of variance ANOVA $p<0.0001$ ), with significant Bonferroni corrected pair-wise differences between $\mathrm{HC}$ versus $\mathrm{AD}(p<0.0001)$ and aMCI versus $\mathrm{AD}$ $(p<0.0001)$

\section{Motion Correction Parameters}

Concerning global accumulated head rotation (Fig. 1), the data did not fulfill the assumption of normal distribution (Shapiro-Wilk normality test) and were thus analyzed using non-parametric Kruskal-Wallis group comparisons and pair-wise Dunn's multiple comparisons tests. There was a significant group difference $(p<0.001)$ as well as significant pair-wise differences between aMCI versus HC $(p<0.05)$ and AD versus HC $(p<0.05)$, as illustrated in Fig. 2a. Receiver operator characteristics (ROC) significantly discriminated $(p<0.01)$ aMCI versus HC with an area under the curve (AUC) of 0.74 (Fig. 3a), as well as AD versus HC ( $p<0.01)$, AUC 0.73 (Fig. 3b). In contrast, there was no significant difference between aMCI versus $\mathrm{AD}$ in accumulated rotation.

Concerning head translation (Fig. 1b), the data did not fulfill the assumption of normal distribution (Shapiro-Wilk normality test). There were equivalent trends as compared to head rotation $(p=0.06)$, yet no significant differences (Fig. 3b).

AD patients had in particular strong rotation along the z-axis (yaw), i.e. rotating the head in a left-right direction like saying "no". We calculated the ratio of z-rotation "no" versus y-rotation (roll, or tilting the head to both sides), as a within-subject normalization (Fig. 2c). These data fulfilled the criteria of normal distribution (ShapiroWilk normality test) and were analyzed using parametric analysis of variance (ANOVA) group comparisons and pair-wise Bonferroni's multiple comparison tests. There was a significant group effect $(p<0.01)$, as well as significant differences between $\mathrm{HC}$ versus $\mathrm{AD}(p<0.05)$ and between aMCI versus $\mathrm{AD}(p<0.05)$.

ROC significantly discriminated $(p<0.05)$ HC versus AD with an AUC of 0.71 (Fig. 4b) and aMCI versus AD $(p<0.05)$ with an AUC of 0.75 . In contrast, this parameter did not discriminate $\mathrm{HC}$ versus aMCI.

\section{Discussion}

The current investigation is based on the observation that head motion artifacts are very common during the neuroimaging workup of neurocognitive decline (Ikari et al. 2012). In agreement with our hypothesis, global head motion was more pronounced in patients and discriminated aMCI and AD from controls. Consequently, head motion not only deteriorates image quality, yet also contains useful discriminatory information. Head motion parameters are routinely estimated during the data processing of fMRI studies (Fox and Greicius 2010), available for free as a "side product" and might complement existing tests to discriminated AD and aMCI from controls. The simple global accumulated head rotation significantly $(p<0.01)$ discriminated aMCI versus $\mathrm{HC}$ with an area under the curve (AUC) of 0.74 as well as $\mathrm{AD}$ versus controls $(p<0.01$, AUC 0.73).

The major point of this paper is to demonstrate that head motion not only deteriorates image quality in a random fashion, but rather is convoluted in a systematic fashion with clinical diagnosis. The main strength of the current investigation is the use of the "freely available" motion parameter in fMRI to discriminate aMCI and AD from controls. The simple parameter of accumulated global head rotation discriminated aMCI and AD from controls. 


\section{A ROTATION}
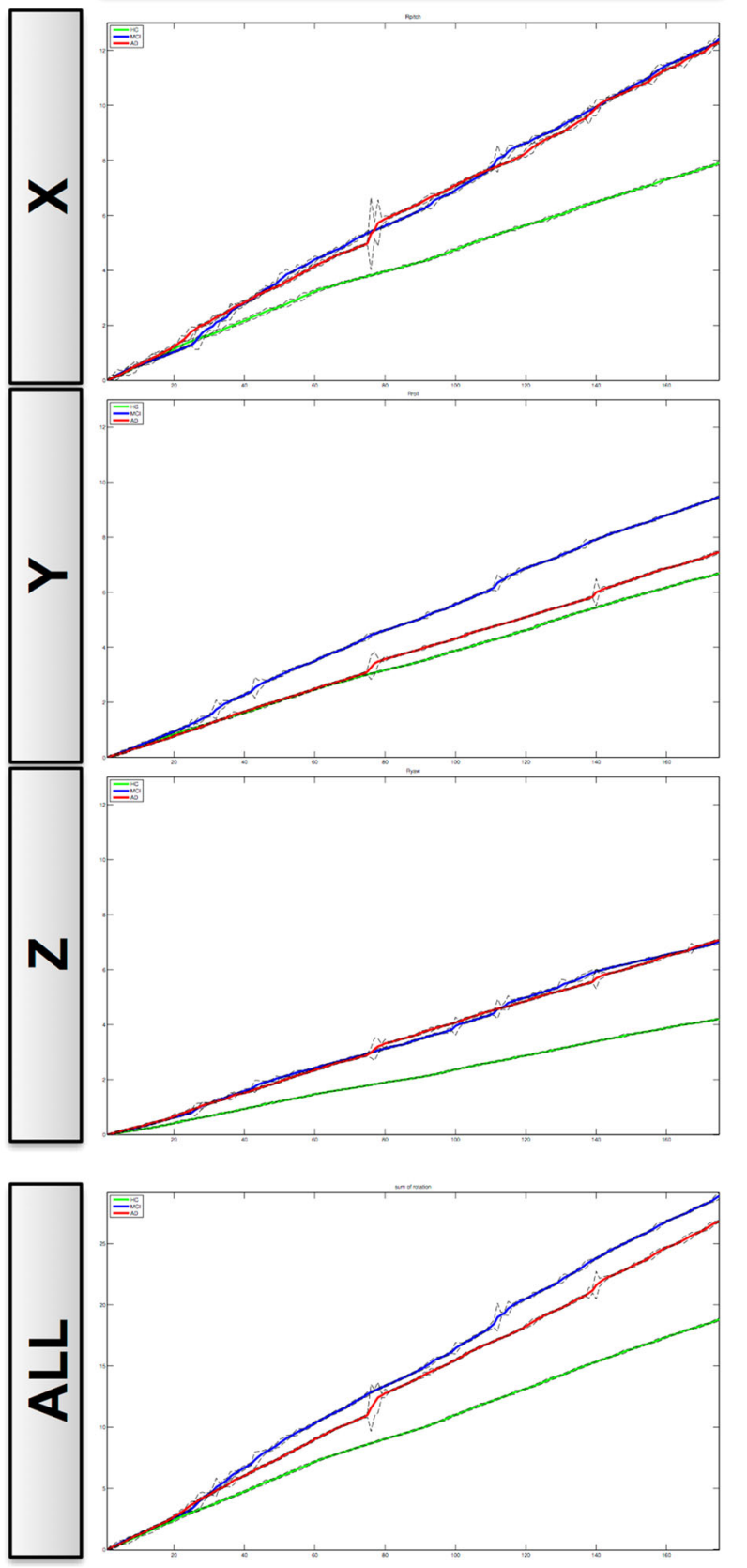

\section{B TRANSLATION}
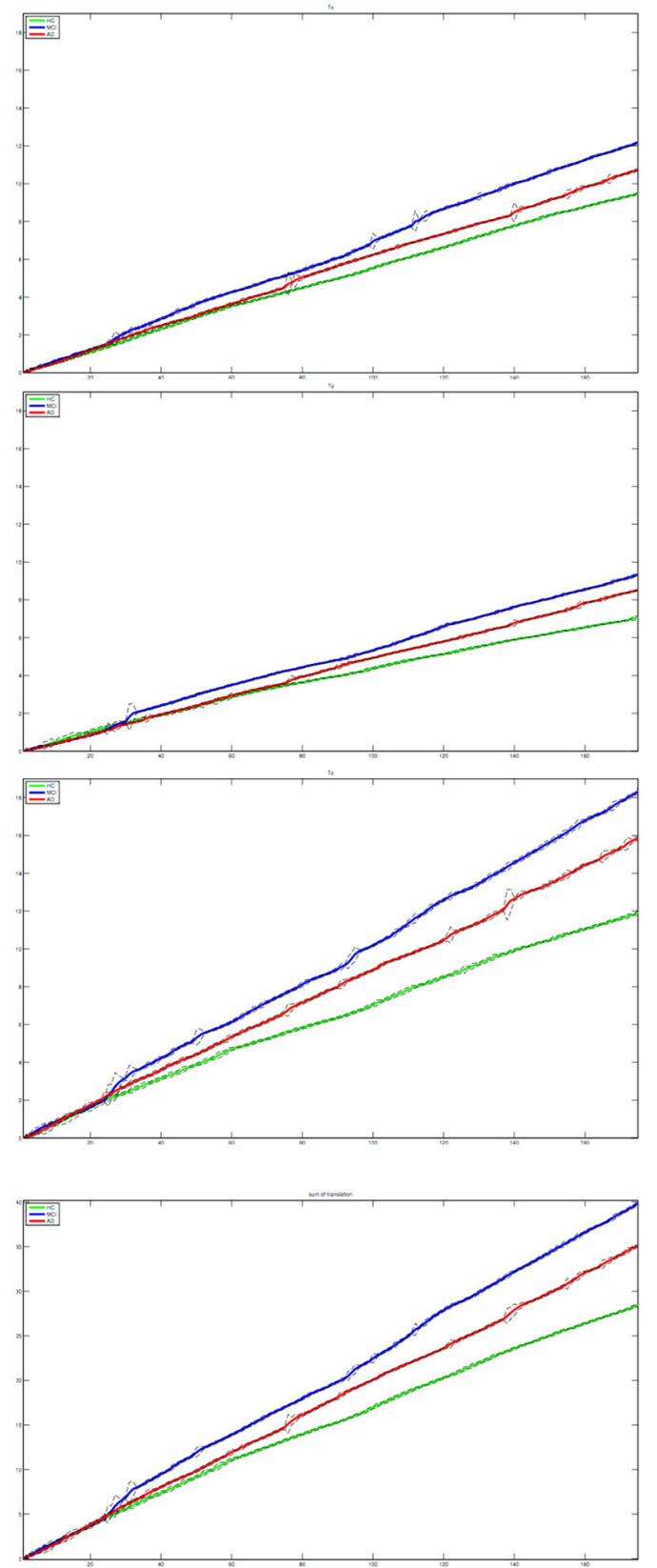

Fig. 1 Average head rotation (a) and translation (b) and standard deviations (black lines) for HC (green), aMCI (blue) and AD (red) separated for $\mathrm{x}, \mathrm{y}, \mathrm{z}$ and average across all 3 axes (Color figure online)

Although these motion parameters are routinely estimated during data processing of fMRI data, these parameters are typically not specifically analyzed. Given this systematic effect, adding motion parameters as an additional covariate in the analysis of fMRI data may be prudent.

Our observation of increased head motion in aMCI and $\mathrm{AD}$ in $\mathrm{FMRI}$ is in agreement with previous experience in the field of fMRI (Sperling 2011) and positron emission tomography (PET) scans, for example in the Japanese Alzheimer disease neuroimaging initiative (J-ADNI) multicenter study (Ikari et al. 2012). Interestingly, a recent investigation demonstrated that head motion produces substantial resting-state fMRI changes despite compensatory spatial registration and regression of motion estimates (Power et al. 2012). In particular, long-distance correlations are decreased while short-distance correlations are 

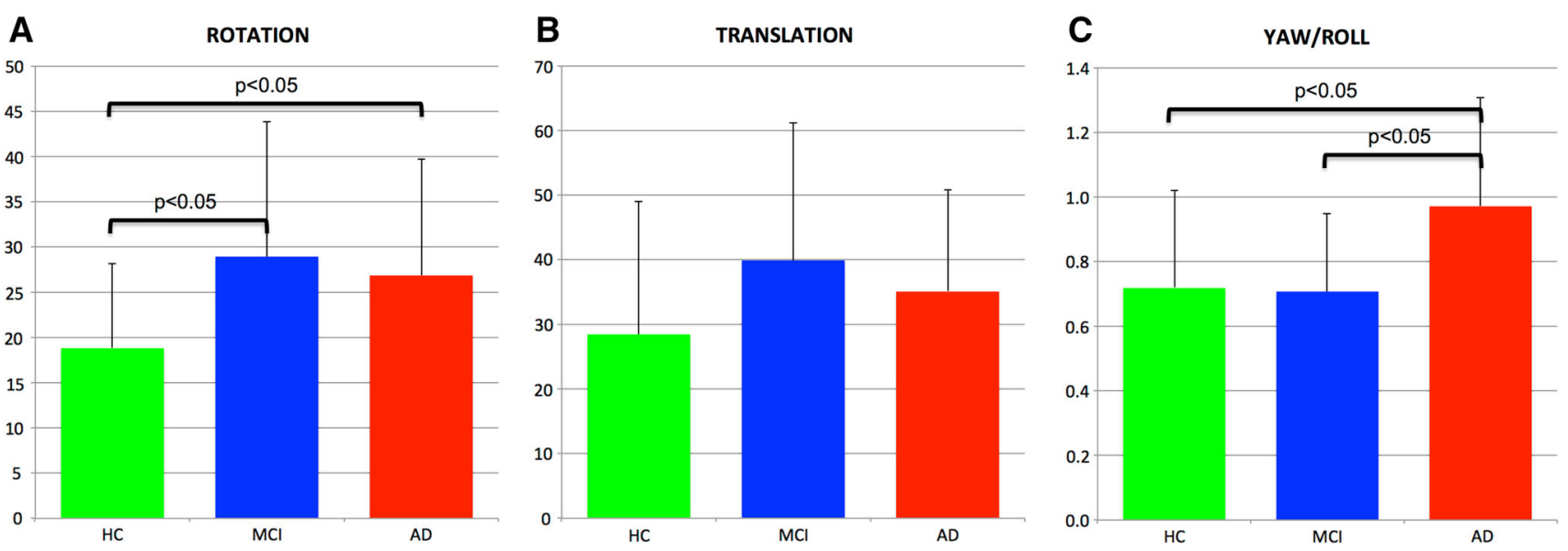

Fig. 2 Illustrates the group average head motion parameters for rotation (a), translation (b) as well as the ratio of yaw (rotation $\mathrm{z}$ ) versus roll (rotation y) in (c). Head rotation discriminated aMCI

(blue) from $\mathrm{HC}$ (green, $p<0.05)$ and $\mathrm{AD}($ red $)$ from $\mathrm{HC}(p<0.05)$. The ration of yaw/roll discriminated $\mathrm{AD}$ from $\mathrm{HC}(p<0.05)$ and $\mathrm{AD}$ from aMCI $(p<0.05)$ (Color figure online)
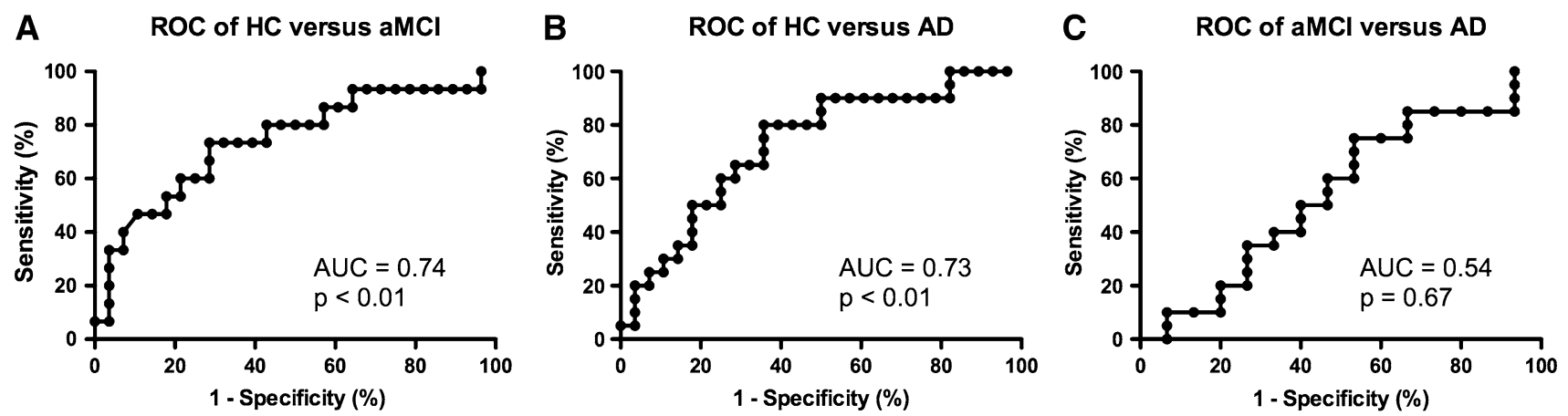

Fig. 3 The receiver operator characteristics (ROC) analysis of the head rotation discriminated aMCI from $\mathrm{HC}(p<0.01$, a) and AD from HC $(p<0.01, \mathbf{b})$, while there was no significant difference between aMCI and AD (c)
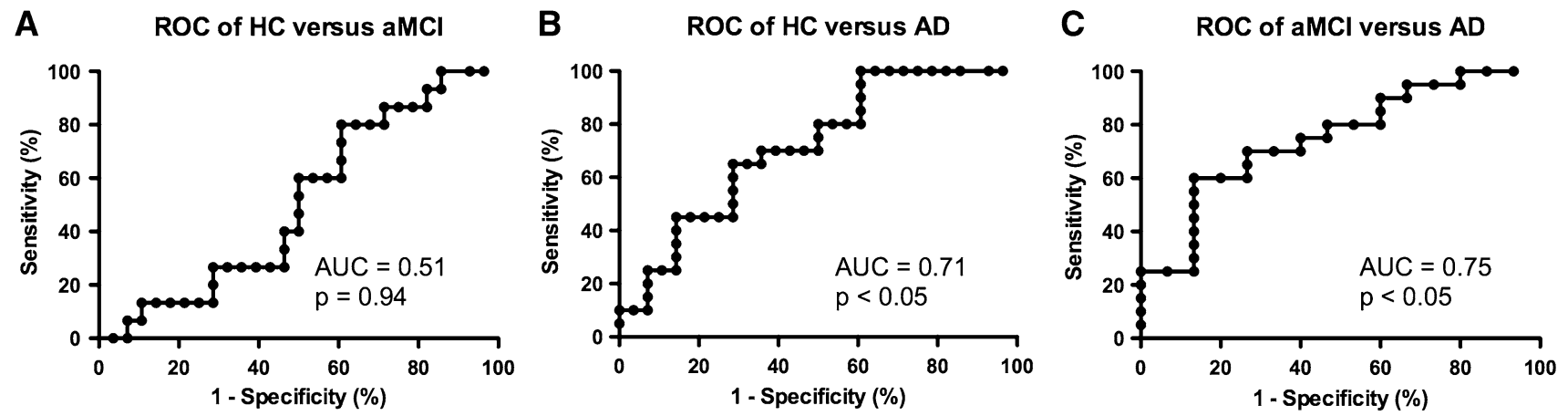

Fig. 4 The ROC analysis of the ratio of yaw/roll discriminated AD versus $\mathrm{HC}(p<0.05$, b) and AD versus aMCI $(p<0.05$, c) but not $\mathrm{HC}$ versus aMCI (a)

increased by head motion. As we observed systematic difference in the amplitude of head motion between the study groups, this implies that group differences in head motion might actually represent a systematic confound for rs-fMRI connectivity studies between controls, MCI and AD subjects.
Due to the spatial constraints of the MRI table and the head coil, some head motions are easier than others. In particular, rotatory movements to the left and right (yaw, like saying "no") and rotation up and down (pitch, like saying "yes") are relatively easy, while left—right tilting movements (roll, inclining the head sideways) or 
longitudinal translation movements are more restricted. In agreement with these spatial constraints, rotation movements were more pronounced than linear translation movements in our data. As discussed above, the discrimination between aMCI versus controls is the clinically most relevant parameter. Concerning the discrimination of $\mathrm{AD}$ versus controls, the clinical diagnosis is oftentimes already established in this advanced stage of the disease. The discrimination between aMCI and AD was less evident in the current data as both groups show considerable head motion. In our sample, AD patients performed in particular strong motion along the $\mathrm{Z}$ axis, i.e. right-left rotation of the head or "no" movements. To relatively normalize this "no" motion per subject, we calculated the ratio between $\mathrm{Z}$ rotation versus $\mathrm{Y}$ rotation (roll or tilting the head sideways). This parameter discriminated AD patients from controls $(p<0.05)$ as well as from aMCI $(p<0.05)$.

\section{Limitations}

Major limitations of the current investigations include the relatively small sample sizes per group, which is due to the strict pre-selection of patients in a single-center study. Our results should thus be confirmed in larger scale studies. Moreover, the current investigation deliberately assessed basic and simple global head movement. The exact origin of the head motions are not assessed in detail in the current investigation and may include decreased compliance, increased anxiety, loss of fine motor coordination and other factors. Future studies might determine whether more detailed motion derived parameters such as speed of acceleration/deceleration or frequency of oscillations may also discriminate between different diseases, for example other forms of dementia such as frontotemoral lobar degeneration or other neurodegenerative diseases such as Parkinson Disease taking into account that head motion might be influenced by multiple factors including for example medication.

\section{Conclusions}

The higher amount of motion in aMCI and AD as compared to controls might be a potential confounding factor in fMRI group comparisons. Moreover, head motion during functional imaging of cognitive decline not only deteriorates image quality but also contains useful discriminatory information. Motion correction parameters are available for free as "side product" during the data preprocessing in fMRI studies and may complement other parameters to discriminate aMCI and AD from controls. More detailed motion parameters might contain specific information in various diseases, which remains to be elucidated in larger scale studies.

Acknowledgments We thank all volunteers and patients for participating in this study. This study was supported, in part, by a grant of the VELUX Foundation

Funding The funders had no role in study design, data collection and analysis, decision to publish, or preparation of the manuscript. No current external funding sources for this study.

\section{References}

Duara R, Barker W, Loewenstein D, Bain L (2009) The basis for disease-modifying treatments for Alzheimer's disease: the sixth annual mild cognitive impairment symposium. Alzheimers Dement 5:66-74

Fan Y, Batmanghelich N, Clark CM, Davatzikos C (2008) Spatial patterns of brain atrophy in MCI patients, identified via highdimensional pattern classification, predict subsequent cognitive decline. Neuroimage 39:1731-1743

Fazekas F, Chawluk JB, Alavi A, Hurtig HI, Zimmerman RA (1987) MR signal abnormalities at $1.5 \mathrm{~T}$ in Alzheimer's dementia and normal aging. AJR Am J Roentgenol 149:351-356

Forlenza OV, Diniz BS, Nunes PV, Memoria CM, Yassuda MS, Gattaz WF (2009) Diagnostic transitions in mild cognitive impairment subtypes. Int Psychogeriatr 21:1088-1095

Fox MD, Greicius M (2010) Clinical applications of resting state functional connectivity. Front Syst Neurosci 4:19

Haller S, Bartsch A, Nguyen D, Rodriguez C, Emch J, Gold G, Lovblad KO, Giannakopoulos P (2010a) Cerebral Microhemorrhage and Iron Deposition in Mild Cognitive Impairment: susceptibility-weighted MR Imaging Assessment. Radiology 257:764-773

Haller S, Nguyen D, Rodriguez C, Emch J, Gold G, Bartsch A, Lovblad KO, Giannakopoulos P (2010b) Individual prediction of cognitive decline in mild cognitive impairment using support vector machine-based analysis of diffusion tensor imaging data. J Alzheimers Dis 22:315-327

Haller S, Missonnier P, Herrmann FR, Rodriguez C, Deiber MP, Nguyen D, Gold G, Lovblad KO, Giannakopoulos P (2013) Individual classification of mild cognitive impairment subtypes by support vector machine analysis of white matter DTI. AJNR Am J Neuroradiol 34:283-291

Holmes C, Boche D, Wilkinson D, Yadegarfar G, Hopkins V, Bayer A, Jones RW, Bullock R, Love S, Neal JW, Zotova E, Nicoll JA (2008) Long-term effects of Abeta42 immunisation in Alzheimer's disease: follow-up of a randomised, placebo-controlled phase I trial. Lancet 372:216-223

Ikari Y, Nishio T, Makishi Y, Miya Y, Ito K, Koeppe RA, Senda M (2012) Head motion evaluation and correction for PET scans with 18F-FDG in the Japanese Alzheimer's disease neuroimaging initiative (J-ADNI) multi-center study. Ann Nucl Med 26:535-544

Lannfelt L, Blennow K, Zetterberg H, Batsman S, Ames D, Harrison J, Masters CL, Targum S, Bush AI, Murdoch R, Wilson J, Ritchie CW (2008) Safety, efficacy, and biomarker findings of PBT2 in targeting Abeta as a modifying therapy for Alzheimer's disease: a phase IIa, double-blind, randomised, placebo-controlled trial. Lancet Neurol 7:779-786

Mariani E, Monastero R, Mecocci P (2007) Mild cognitive impairment: a systematic review. J Alzheimers Dis 12:23-35 
McKhann G, Drachman D, Folstein M, Katzman R, Price D, Stadlan EM (1984) Clinical diagnosis of Alzheimer's disease: report of the NINCDS-ADRDA work group under the auspices of department of health and human services task force on Alzheimer's disease. Neurology 34:939-944

Misra C, Fan Y, Davatzikos C (2009) Baseline and longitudinal patterns of brain atrophy in MCI patients, and their use in prediction of short-term conversion to AD: results from ADNI. Neuroimage 44:1415-1422

Monsch AU, Kressig RW (2010) Specific care program for the older adults: memory clinics. Eur Geriatr Med 1:128-131

Mueller S, Keeser D, Reiser MF, Teipel S, Meindl T (2012) Functional and structural $\mathrm{mr}$ imaging in neuropsychiatric disorders, part 1: imaging techniques and their application in mild cognitive impairment and Alzheimer disease. AJNR Am J Neuroradiol 33:1845-1850

Nitsch RM, Hock C (2008) Targeting beta-amyloid pathology in Alzheimer's disease with Abeta immunotherapy. Neurotherapeutics 5:415-420

O'Dwyer L, Lamberton F, Bokde AL, Ewers M, Faluyi YO, Tanner C, Mazoyer B, O'Neill D, Bartley M, Collins DR, Coughlan T, Prvulovic D, Hampel H (2012) Using support vector machines with multiple indices of diffusion for automated classification of mild cognitive impairment. PLoS ONE 7:e32441
Petersen RC (2004) Mild cognitive impairment as a diagnostic entity. J Intern Med 256:183-194

Petersen RC, Negash S (2008) Mild cognitive impairment: an overview. CNS Spectr 13:45-53

Plant C, Teipel SJ, Oswald A, Bohm C, Meindl T, Mourao-Miranda J, Bokde AW, Hampel H, Ewers M (2010) Automated detection of brain atrophy patterns based on MRI for the prediction of Alzheimer's disease. Neuroimage 50:162-174

Power JD, Barnes KA, Snyder AZ, Schlaggar BL, Petersen SE (2012) Spurious but systematic correlations in functional connectivity MRI networks arise from subject motion. Neuroimage 59:2142-2154

Sperling R (2011) Potential of functional MRI as a biomarker in early Alzheimer's disease. Neurobiol Aging 32(Suppl 1):S37-S43

Winblad B, Palmer K, Kivipelto M, Jelic V, Fratiglioni L, Wahlund LO, Nordberg A, Backman L, Albert M, Almkvist O, Arai H, Basun H, Blennow K, de Leon M, DeCarli C, Erkinjuntti T, Giacobini E, Graff C, Hardy J, Jack C, Jorm A, Ritchie K, van Duijn C, Visser P, Petersen RC (2004) Mild cognitive impairment-beyond controversies, towards a consensus: report of the International Working Group on Mild Cognitive Impairment. J Intern Med 256:240-246 